\title{
Color-kinematics duality from the Bern-Kosower formalism
}

\author{
Naser Ahmadiniaz, ${ }^{1, *}$ Filippo Maria Balli $\odot,{ }^{2, \dagger}$ Cristhiam Lopez-Arcos $\odot,{ }^{3, \ddagger}$ \\ Alexander Quintero Vélez, ${ }^{3, \S}$ and Christian Schubert $\oplus^{4, \|}$ \\ ${ }^{1}$ Helmholtz-Zentrum Dresden-Rossendorf, Bautzner Landstraße 400, 01328 Dresden, Germany \\ ${ }^{2}$ Dipartimento di Scienze Fisiche, Informatiche e Matematiche, Università degli Studi di Modena e \\ Reggio Emilia, Via Campi 213/A, I-41125 Modena, Italy and INFN, Sezione di Bologna, \\ Via Irnerio 46, I-40126 Bologna, Italy \\ ${ }^{3}$ Escuela de Matemáticas, Universidad Nacional de Colombia Sede Medellín, \\ Carrera 65 \# 59A-110, Medellín, Colombia \\ ${ }^{4}$ Instituto de Física y Matemáticas Universidad Michoacana de San Nicolás de Hidalgo Edificio C-3, \\ Apdo. Postal 2-82 C.P. 58040, Morelia, Michoacán, México
}

(Received 25 May 2021; accepted 8 July 2021; published 6 August 2021)

\begin{abstract}
We present a novel procedure to construct Yang-Mills Berends-Giele currents from the Bern-Kosower formalism for one-loop gluon amplitudes. The obtained currents naturally obey color-kinematics duality. As a feedback to the Bern-Kosower formalism we outline how the multiparticle fields can be used to significantly streamline the pinch procedure for the reducible one-loop amplitudes.
\end{abstract}

DOI: 10.1103/PhysRevD.104.L041702

\section{INTRODUCTION}

In non-Abelian gauge theory, the nonlinearity of the Yang-Mills gauge transformations tends to make it difficult to write $n$-gluon amplitudes in a way that would make the gauge Ward identities transparent (see [1] and references therein). On shell they imply transversality of the corresponding $n$-gluon matrix elements, that is their vanishing under the replacement of any gluon polarization vector $\varepsilon_{i}^{\mu}$ by the corresponding momentum $k_{i}^{\mu}$. In the standard Feynman diagram approach this involves intricate cancellations between one-particle irreducible diagrams and reducible diagrams that have trees sewn onto loops. Such trees, with any number of gluons on shell and one off shell (sewn onto the loop) are called Berends-Giele currents. Berends and Giele already introduced the idea of interpreting them in terms of multigluon states [2]. More recently, it was found that these currents can be written in a certain gauge, the so-called Bern-CarrascoJohansson (BCJ) gauge [3], motivated by a result of Bern, Carrasco, and Johansson that allows one to write the

\footnotetext{
*n.ahmadiniaz@hzdr.de

†filippo.balli@unimore.it

\$cmlopeza@unal.edu.co

\$aquinte2@unal.edu.co

sschubert@ifm.umich.mx
}

Published by the American Physical Society under the terms of the Creative Commons Attribution 4.0 International license. Further distribution of this work must maintain attribution to the author(s) and the published article's title, journal citation, and DOI. Funded by SCOAP. numerators for the color ordered amplitudes in such a way that these obey the same relations as their color factors [4]. This has come to be known as color-kinematics duality. At the level of the Berends-Giele currents, the duality is made explicit by means of some generalized Jacobi identities (GJI), as has been shown by Mafra and Schlotterer [5]. They arrived at these identities by carefully looking at the structure of multiparticle vertex operators for ten dimensional Super Yang-Mills (SYM), making use of the technology of pure spinor Becchi-Rouet-Stora-Tyutin (BRST) cohomology.

The study of the Berends-Giele currents has recently received a good deal of attention [3,6-12], since writing amplitudes in this BCJ form is the key to exhibiting color-kinematics as well as double-copy duality [13,14]. However, their construction quickly becomes tedious in practice as the number of legs grows, and sophisticated methods have been proposed to deal with this problem (see e.g., [3,11] for an approach involving highly nonlinear gauge transformations resembling pure spinor BRST transformations).

In this paper, we will use the Bern-Kosower formalism to develop a simple and direct method to construct the currents in the BCJ gauge. This formalism was originally derived using the field-theory limit of string amplitudes [15-17], and led to a set of rules that allows one to directly write down Feynman-Schwinger type parameter integrals for the one-loop on-shell $n$-gluon matrix elements. For our present purposes, the most relevant aspect of these BernKosower rules is that they allow one to reconstruct the integrands of the reducible contributions to the matrix elements from the one of the irreducible one by a pinching 
procedure. The application of the Bern-Kosower pinch rules requires one to first perform certain partial integrations to the integrand that effectively remove quartic vertices. The general structure of the resulting integrands was studied by Strassler [18,19] in the framework of the worldline formalism, an alternative approach to perturbation theory that to some extent mimics string perturbation theory (for reviews see [20,21]). He found that the partial integration procedure naturally leads to the appearance of "Lorentz cycles" $Z_{k}\left(i_{1}, i_{2}, \ldots, i_{k}\right)$, defined as the traces of products of gluon field strength tensors $f_{i}^{\mu \nu} \equiv k_{i}^{\mu} \varepsilon_{i}^{\nu}-\varepsilon_{i}^{\mu} k_{i}^{\nu}$. This fact has already played an important role in various applications of the worldline formalism [22-25].

Clearly, the pinching procedure must hold the full information on the Berends-Giele currents attached to the loop. Our present work is motivated by the observation that it leads to the appearance of generalized structures now also involving multiparticle fields. We observe that the pinching procedure naturally generates these multiparticle fields in the BCJ gauge.

Notation: For a word $I=i_{1} i_{2} \ldots i_{p}$ we set $|I|=p$. We also have multiparticle momentum $k_{I}^{\mu}=k_{i_{1}}^{\mu}+k_{i_{2}}^{\mu}+\cdots+$ $k_{i_{p}}^{\mu}$ and Mandelstam variables $s_{I}=k_{I}^{2}$. We write Lorentz cycles involving multiparticle fields numerators as

$$
Z_{k}\left(I_{1}, \ldots, I_{k}\right)=\left(\frac{1}{2}\right)^{\delta_{k 2}} \operatorname{tr}\left(\prod_{i=1}^{k} f_{I_{i}}\right)
$$

\section{SYMMETRIC PARTIAL INTEGRATION AND CYCLE DECOMPOSITION}

Central to the Bern-Kosower formalism is the following master formula for the color-ordered one-loop $n$-gluon correlator with a scalar loop,

$$
\begin{aligned}
\Gamma\left(k_{1}, \varepsilon_{1} ; \ldots ; k_{n}, \varepsilon_{n}\right)= & (-i g)^{n} \operatorname{tr}\left(T^{a_{1}} \cdots T^{a_{n}}\right) \int_{0}^{\infty} d T(4 \pi T)^{-\frac{D}{2}} \mathrm{e}^{-m^{2} T} \int_{0}^{T} d \tau_{1} \int_{0}^{\tau_{1}} d \tau_{2} \cdots \int_{0}^{\tau_{n-2}} d \tau_{n-1} \\
& \times\left.\exp \left\{\sum_{i, j=1}^{n}\left(\frac{1}{2} G_{i j} k_{i} \cdot k_{j}-i \dot{G}_{i j} \varepsilon_{i} \cdot k_{j}+\frac{1}{2} \ddot{G}_{i j} \varepsilon_{i} \cdot \varepsilon_{j}\right)\right\}\right|_{\varepsilon_{1} \ldots \varepsilon_{n}} .
\end{aligned}
$$

Here $G_{i j} \equiv G\left(\tau_{i}, \tau_{j}\right)$ are the worldline Green's function $G\left(\tau, \tau^{\prime}\right)=\left|\tau-\tau^{\prime}\right|-\frac{\left(\tau-\tau^{\prime}\right)^{2}}{T}$, with an antisymmetric derivative $\left(\dot{G}_{i j}=-\dot{G}_{j i}\right)$. For a given $n$, we expand the exponential keeping only the linear terms in each of the polarization vectors $\varepsilon_{1}, \ldots, \varepsilon_{n}$. The resulting integrand is of the form

$$
\left.\exp \{\cdot\}\right|_{\varepsilon_{1} \varepsilon_{2} \ldots \varepsilon_{n}} \equiv(-i)^{n} P_{n}\left(\dot{G}_{i j}, \ddot{G}_{i j}\right) \mathrm{e}^{\frac{1}{2} \sum_{i, j=1}^{n} G_{i j} k_{i} \cdot k_{j}}
$$

with polynomials $P_{n}$.

As it stands, (2) represents the off-shell irreducible vertex function induced by a scalar loop. To obtain the corresponding on-shell matrix elements, one normally would have to perform a Legendre transformation, which diagrammatically means attaching trees to the loop in all possible ways. Alternatively, in the Bern-Kosower formalism these one-particle reducible contributions can be included by the following pinching procedure:

(i) Remove all second derivatives $\ddot{G}_{i j}$ contained in $P_{n}$ through integration by parts.

(ii) Draw all possible $\phi^{3}$ one-loop diagrams $D_{i}$ with $n$ legs, labeled $1, \ldots, n$ (following the ordering of the color trace).

(iii) A diagram will contribute if and only if each vertex except the ones attached to the loop corresponds to a possible pinch. A vertex with labels $i<j$ can be pinched if and only if $P_{n}$ is linear in $\dot{G}_{i j}$. The pinching replaces this $\dot{G}_{i j}$ by a factor of $1 / s_{i j}$, removes the vertex and transfers the label $i$ to the ingoing leg (see Fig. 1).

The $\tau_{j}$ integration is omitted and the index $j$ replaced by $i$ in all $G_{k l}, \dot{G}_{k l}$. The trees are to be pruned recursively starting with the outermost vertices. For details and examples see [17,20].

The result of the integration by parts procedure is ambiguous starting from the four-gluon case [19]. For our present purposes, it is imperative to fix this ambiguity in a way that preserves the full permutation symmetry of the master formula (2). A suitable "symmetric partial integration" algorithm was proposed in [26]. It transforms the polynomial $P_{n}\left(\dot{G}_{i j}, \ddot{G}_{i j}\right)$ into a polynomial $Q_{n}\left(\dot{G}_{i j}\right)$ that permits a cycle decomposition into terms with a definite cycle content, each cycle corresponding to a product

$\dot{G}\left(i_{1}, i_{2}, \ldots, i_{k}\right)=\dot{G}_{i_{1} i_{2}} \dot{G}_{i_{2} i_{3}} \cdots \dot{G}_{i_{k} i_{1}} Z_{k}\left(i_{1}, i_{2}, \ldots, i_{k}\right)$

with $Z_{k}\left(i_{1}, i_{2}, \ldots, i_{k}\right)$ like in (1). These cycles are invariant under the operations of cyclic permutations and inversion. Apart from cycle factors, a term may have a leftover called

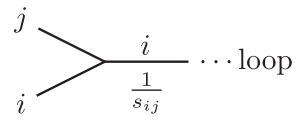

FIG. 1. Pinching of a vertex according to the Bern-Kosower rules. 
a "tail", and is denoted by $T\left(i_{1}, i_{2}, \ldots, i_{k}\right)$ where $i_{1}, i_{2}, \ldots, i_{k}$ are the indices not yet bound up in any cycle. Although the tails are not manifestly transversal, they turn into total derivatives whenever any of the $\varepsilon_{i_{m}}$ contained in them is replaced by $k_{i_{m}}$. For example, the cycle decompositions of $Q_{3}$ and $Q_{4}$ read

$$
\begin{aligned}
& Q_{3}=Q_{3}^{3}+Q_{3}^{2} \\
& Q_{3}^{3}=\dot{G}(1,2,3) \\
& Q_{3}^{2}=\dot{G}(1,2) T(3)+\dot{G}(2,3) T(1)+\dot{G}(3,1) T(2) \\
Q_{4}= & Q_{4}^{4}+Q_{4}^{3}+Q_{4}^{2}+Q_{4}^{22} \\
Q_{4}^{4}= & \dot{G}(1,2,3,4)+\dot{G}(1,2,4,3)+\dot{G}(1,3,2,4) \\
Q_{4}^{3}= & \dot{G}(1,2,3) T(4)+\dot{G}(2,3,4) T(1)+\dot{G}(3,4,1) T(2) \\
& +\dot{G}(4,1,2) T(3) \\
Q_{4}^{2}= & \dot{G}(1,2) T(3,4)+\dot{G}(1,3) T(2,4)+\dot{G}(1,4) T(2,3) \\
& +\dot{G}(2,3) T(1,4)+\dot{G}(2,4) T(1,3)+\dot{G}(3,4) T(1,2) \\
Q_{4}^{22}= & \dot{G}(1,2) \dot{G}(3,4)+\dot{G}(1,3) \dot{G}(2,4)+\dot{G}(1,4) \dot{G}(2,3),
\end{aligned}
$$

where the cycle content of a term is indicated by the superscript. At this level, only the one and two tails appear,

$$
\begin{aligned}
T(a) \equiv & \sum_{r} \dot{G}_{a r} \varepsilon_{a} \cdot k_{r} \\
T(a, b) \equiv & \sum_{\substack{r, s \\
(r, s) \neq(b, a)}} \dot{G}_{a r} \varepsilon_{a} \cdot k_{r} \dot{G}_{b s} \varepsilon_{b} \cdot k_{s} \\
& +\frac{1}{2} \dot{G}_{a b} \varepsilon_{a} \cdot \varepsilon_{b}\left[\sum_{r \neq b} \dot{G}_{a r} k_{a} \cdot k_{r}-\sum_{s \neq a} \dot{G}_{b s} k_{b} \cdot k_{s}\right] .
\end{aligned}
$$

In the two tail, note the exclusion of terms from the sums that would correspond to the appearance of a new cycle $\dot{G}(a, b)$ in the tail, and thus to an overcounting. Note also that when advancing from the $n$-gluon amplitude to the $(n+1)$-gluon one the only new ingredient to be calculated is the $n-1$ tail; this is a relatively easy task. The integrands $Q_{5}$ and $Q_{6}$ are explicitly shown in Appendix C of [20].

\section{THE STRUCTURE OF WORLDLINE INTEGRANDS AND PINCH OPERATORS}

The main ingredients for our approach are the BernKosower rules [15-17] and the symmetric partial integration [26]. From these we identify two essential objects. First, we have the permutation invariant integrand $Q_{n}$. Second, for two adjacent legs $i$ and $j$ with $i<j$, we have an antisymmetric pinch operator acting on $Q_{n}$ as

$$
\mathcal{D}_{i j} Q_{n}=\left.\frac{\partial}{\partial \dot{G}_{i j}} Q_{n}\right|_{\substack{\dot{G}_{i j}=0 \\ \dot{G}_{j k} \rightarrow \dot{G}_{i k}}}
$$

Diagrammatically, this corresponds to pinching the two adjacent legs $i$ and $j$ (see Fig. 1).

In order to understand the basic link between the former and the GJI, let us first define what they are. Consider the free Lie algebra, $\operatorname{Lie}[1, \ldots, n]$, generated by all words with letters in $1, \ldots, n$ (see e.g., [27,28]). Moreover, let $\ell$ be the left to right bracketing on $\operatorname{Lie}[1, \ldots, n]$, which is defined recursively by

$$
\begin{aligned}
\ell\left(i_{1} i_{2} \cdots i_{k}\right) & =\ell\left(i_{1} i_{2} \cdots i_{k-1}\right) i_{k}-i_{k} \ell\left(i_{1} i_{2} \cdots i_{k-1}\right), \\
\ell(i) & =i \\
\ell(\oslash) & =0 .
\end{aligned}
$$

Then the GJI of order $k$ can be characterized as the set of identities in $\operatorname{Lie}[1, \ldots, n]$ of the form

$$
I \ell(J)+J \ell(I)=0,
$$

for every pair of nonempty words $I$ and $J$ such that $|I|+|J|=k$.

Now that we have the GJI, we can explore the connection between these and the pinch operators in more detail. For this, we need to understand a little better the structure of $Q_{n}$. Given a map $\alpha:\{1, \ldots, n\} \rightarrow\{1, \ldots, n\}$, consider the following polynomial of degree $n$ on the $\dot{G}_{i j}$ 's

$$
Q_{n}^{(\alpha)}=\sum_{\text {perm }} c_{12 \ldots n} \dot{G}_{1 \alpha(1)} \dot{G}_{2 \alpha(2)} \cdots \dot{G}_{n \alpha(n)}
$$

where the coefficients depend on the polarizations and momenta. Then, $Q_{n}$ can be written as a sum of polynomials of this form. Therefore, to understand how the pinch operators act on $Q_{n}$, it will be enough to consider their action on such polynomials. For this, it will be convenient first to examine an specific example. Take $n=4$ and $\alpha$ such that $\alpha(1)=1, \alpha(2)=1, \alpha(3)=2$ and $\alpha(4)=3$. By a straightforward calculation one finds that the action of the pinch operator $\mathcal{D}_{12}$ on the resulting polynomial $Q_{4}^{(\alpha)}$ yields the polynomial

$$
\begin{aligned}
& \left(c_{3142}-c_{3241}\right) \dot{G}_{13}^{2} \dot{G}_{14}+\left(c_{4312}-c_{4321}\right) \dot{G}_{13} \dot{G}_{34}^{2} \\
& \quad+(3 \leftrightarrow 4) .
\end{aligned}
$$

We can check directly that each of the coefficients is antisymmetric in 1 and 2, i.e., they satisfy the GJI of order 1. Let us next apply the pinch operator $\mathcal{D}_{13}$ to (11). The result is

$$
\left(c_{4132}-c_{4231}+c_{4312}-c_{4321}\right) \dot{G}_{14}^{2}
$$


Now the coefficient satisfies the Jacobi identity in 1, 2 and 3 , i.e., the GJI of order 2.

Returning to the general case, we may infer that the iterated action of the pinch operators $\mathcal{D}_{12}, \mathcal{D}_{13}, \ldots, \mathcal{D}_{1(n-1)}$ on a polynomial of the form (10) will produce a monomial. Explicitly,

$$
\mathcal{D}_{1(n-1)} \cdots \mathcal{D}_{13} \mathcal{D}_{12} Q_{n}^{(\alpha)}=\tilde{c}_{12 \cdots n} \dot{G}_{1 n}^{2},
$$

where the coefficient $\tilde{c}_{12 \cdots n}$ satisfies the GJI of order $n-1$ in $1,2, \ldots, n-1$. We have checked that this is the case up to degree $n=9$.

We make a final remark on how the above can be represented diagrammatically. To this end, one just have to remark that a left to right bracketing in $\operatorname{Lie}[1, \ldots, n]$ can be interpreted as a planar binary tree and vice versa. For example,

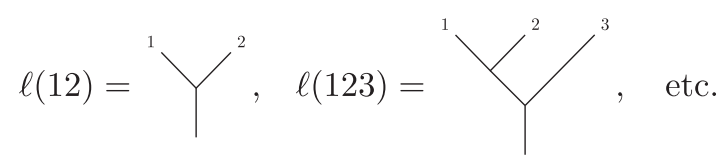

Using this notation, we find, for instance, that the iterated action of $\mathcal{D}_{1,2}$ and $\mathcal{D}_{1,3}$ on $Q_{n}$ can be graphically represented as

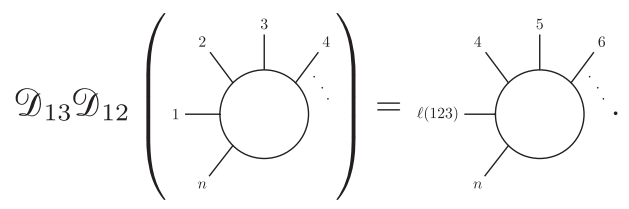

\section{MULTIPARTICLE FIELDS FROM PINCHING}

The properties of the Bern-Kosower integrands discussed in the previous section allow us to build the Berends-Giele currents for Yang-Mills directly in the BCJ gauge. The tree propagators could be obtained by the conventional Bern-Kosower pinch rules, but we prefer to exploit the symmetries in the numerators that allow one to obtain all the numerators of the Berends-Giele currents from a single calculation, by relabeling the legs and taking linear combinations where necessary. For the propagators we can make use of the inverse of the Kawai-Lewellen-Tye (KLT) matrix [13,29,30], which was given a Berends-Giele description in [31,32] (see also [33]).

The appropriate polynomial for finding the field strength numerator is just the sum of the terms in $Q_{n}$ with a single one-cycle component $\tilde{Q}_{n}=Q_{n}^{2}+Q_{n}^{3}+\cdots+Q_{n}^{n}$. Using (13) we obtain

$$
\begin{aligned}
\mathcal{D}_{1(n-1)} \cdots \mathcal{D}_{13} \mathcal{D}_{12} \tilde{Q}_{n} & =Z_{2}(12 \ldots n-1, n) \dot{G}_{1 n}^{2} \\
& =\frac{1}{2} f_{12 \cdots(n-1)}^{\mu \nu} f_{n \nu \mu} \dot{G}_{1 n}^{2},
\end{aligned}
$$

where $f_{12 \cdots(n-1)}^{\mu \nu}$ satisfy the GJI.

From the field-strength tensors one can also extract the multiparticle polarizations, but it turns out that those can alternatively be obtained applying pinch operators just to the tails

$$
\begin{aligned}
& \mathcal{D}_{1(n-2)} \cdots \mathcal{D}_{13} \mathcal{D}_{12} T(1,2, \ldots, n-2) \\
& \quad=\varepsilon_{12 \cdots(n-2)} \cdot k_{n-1} \dot{G}_{1(n-1)}+\varepsilon_{12 \cdots(n-2)} \cdot k_{n} \dot{G}_{1 n} .
\end{aligned}
$$

The multiparticle polarizations obtained in either way will satisfy the corresponding GJI. Note, however, that the second tail-pinching method requires one to know the tails to one order higher than is necessary for the first cyclepinching approach.

\section{EXAMPLES}

We will now work out the currents up to $n=5$. We start with $Q_{2}$ which has no trees and therefore no derivatives, but leads to the first solution

$$
Z_{2}(1,2) \dot{G}_{12}^{2}
$$

Comparing (17) with (1) one arrives at

$$
Z_{2}(1,2)=\frac{1}{2} f_{1}^{\mu \nu} f_{2 \nu \mu},
$$

which gives us just the usual Abelian one-particle field strength tensor $f_{i}^{\mu \nu}=k_{i}^{\mu} \varepsilon_{i}^{\nu}-k_{i}^{\nu} \varepsilon_{i}^{\mu}$ that was already introduced above. This is also the Berends-Giele current for this case.

\section{A. Two-particle case}

This numerator is extracted from the $Q_{3}$ integrand (5). To obtain the external-leg bubble integrand numerator we only have to pinch two legs

$$
Q_{3}^{(12)}=\mathcal{D}_{12} Q_{3} \text {. }
$$

The explicit expression for the Lorentz two-cycle that goes with $\dot{G}_{13}^{2}$ in (15) for this case is

$Z_{2}(12,3)=\varepsilon_{2} \cdot k_{1} Z_{2}(1,3)-\frac{1}{2} Z_{3}(1,2,3)-(1 \leftrightarrow 2)$.

We can immediately see that $f_{3}^{\nu \mu}$ can be factorized out to give the two-current field strength numerator

$$
f_{12}^{\mu \nu}=\varepsilon_{2} \cdot k_{1} f_{1}^{\mu \nu}-\left(f_{1} f_{2}\right)^{\mu \nu}-(1 \leftrightarrow 2) .
$$


From (7) and (16) we extract the two-particle polarization

$$
\varepsilon_{12}^{\mu}=\frac{1}{2}\left[\varepsilon_{2} \cdot k_{1} \varepsilon_{1}^{\mu}-\varepsilon_{1 \rho} f_{2}^{\rho \mu}-(1 \leftrightarrow 2)\right] .
$$

Evidently $f_{12}^{\mu \nu}$ and $\varepsilon_{12}^{\mu}$ are antisymmetric in 1 and 2 . The Berends-Giele current here is

$$
\mathcal{F}_{12}^{\mu \nu}=\frac{f_{12}^{\mu \nu}}{s_{12}},
$$

which can also be obtained directly from the classical action via perturbiner methods $[31,34,35]$. Such methods will not work at higher points if we look for the BCJ gauge.

\section{B. Three-particle case}

Here $\mathcal{D}_{13} \mathcal{D}_{12} \tilde{Q}_{4}$ will immediately deliver the numerator for the field strength three-current

$$
\begin{aligned}
f_{123}^{\mu \nu}= & k_{123}^{\mu} \varepsilon_{123}^{\nu}-k_{12} \cdot k_{3} \varepsilon_{12}^{\mu} \varepsilon_{3}^{\nu} \\
& -k_{1} \cdot k_{2}\left(\varepsilon_{1}^{\mu} \varepsilon_{23}^{\nu}+\varepsilon_{13}^{\mu} \varepsilon_{2}^{\nu}\right)-(\mu \leftrightarrow \nu) .
\end{aligned}
$$

To get the polarization we use the expression for $T(1,2,3)$ that can be found in the Appendix $\mathrm{C}$ of [20] and by (16)

$$
\begin{aligned}
\varepsilon_{123}^{\mu}= & \frac{1}{2}\left[\left(k_{3} \cdot \varepsilon_{12}\right) \varepsilon_{3}^{\mu}-\left(k_{12} \cdot \varepsilon_{3}\right) \varepsilon_{12}^{\mu}+\varepsilon_{12 \nu} f_{3}^{\nu \mu}\right. \\
& \left.-\varepsilon_{3 \nu} f_{12}^{\nu \mu}\right]-k_{123}^{\mu} h_{123},
\end{aligned}
$$

where

$$
h_{123}=\frac{1}{4} \varepsilon_{1} \cdot \varepsilon_{2} \varepsilon_{3} \cdot\left(k_{2}-k_{1}\right) .
$$

We employ the $h_{I}$ scalars here for notational compactness and to compare with the results of [5], where those were introduced to take the numerators from the Lorenz gauge to the BCJ gauge. The expressions for the $h_{I}$ 's that appear naturally in our method are different from theirs, but the difference can be understood as a gauge transformation. Both (24) and (25) satisfy the GJI

$$
\varepsilon_{123}^{\mu}+\varepsilon_{213}^{\mu}=0, \quad \varepsilon_{123}^{\mu}+\varepsilon_{312}^{\mu}+\varepsilon_{231}^{\mu}=0 .
$$

Now the Berends-Giele current for the polarization looks like

$$
\mathcal{A}_{123}^{\mu}=\frac{\varepsilon_{123}^{\mu}}{s_{12} s_{123}}+\frac{\varepsilon_{321}^{\mu}}{s_{23} s_{123}} .
$$

\section{Four-particle case}

Following the same procedure we arrive at the field strength numerator in the four-particle case

$$
\begin{aligned}
f_{1234}^{\mu \nu}= & k_{1234}^{\mu} \varepsilon_{1234}^{\nu}+k_{123} \cdot k_{4} \varepsilon_{123}^{\nu} \varepsilon_{4}^{\mu}+k_{12} \cdot k_{3}\left[\varepsilon_{12}^{\nu} \varepsilon_{34}^{\mu}\right. \\
& \left.+\varepsilon_{124}^{\nu} \varepsilon_{3}^{\mu}\right]+k_{1} \cdot k_{2}\left[\varepsilon_{1}^{\nu} \varepsilon_{234}^{\mu}+\varepsilon_{134}^{\nu} \varepsilon_{2}^{\mu}+\varepsilon_{13}^{\nu} \varepsilon_{24}^{\mu}\right. \\
& \left.+\varepsilon_{14}^{\nu} \varepsilon_{23}^{\mu}\right]-(\mu \leftrightarrow \nu) .
\end{aligned}
$$

We also find the numerator of the four-particle polarization

$$
\begin{aligned}
\varepsilon_{1234}^{\mu}= & \frac{1}{2}\left[\varepsilon_{4}^{\mu}\left(\varepsilon_{123} \cdot k_{4}\right)-\varepsilon_{123}^{\mu}\left(\varepsilon_{4} \cdot k_{123}\right)+\varepsilon_{123 \nu} f_{4}^{\nu \mu}\right. \\
& \left.-\varepsilon_{4 \nu} f_{123}^{\nu \mu}\right]+\varepsilon_{3}^{\mu}\left(k_{12} \cdot k_{3}\right) h_{124}+k_{1} \cdot k_{2}\left(\varepsilon_{2}^{\mu} h_{134}\right. \\
& \left.-\varepsilon_{1}^{\mu} h_{234}\right)-k_{1234}^{\mu} h_{1234},
\end{aligned}
$$

where

$$
\begin{aligned}
h_{1234}= & \frac{1}{4}\left[\varepsilon_{1} \cdot \varepsilon_{2} \varepsilon_{3} \cdot k_{2} \varepsilon_{4} \cdot\left(k_{1}-k_{23}\right)\right. \\
& \left.+\frac{1}{2}\left(\varepsilon_{1} \cdot \varepsilon_{2} \varepsilon_{3} \cdot \varepsilon_{4} k_{2} \cdot k_{3}\right)-(123 \rightarrow 312)\right] \\
& -(1 \leftrightarrow 2) .
\end{aligned}
$$

A numerator with the BCJ property was also obtained in [5] with a two-step procedure involving a BRST-inspired transformation. The GJI satisfied by these numerators are

$$
\begin{gathered}
f_{1234}^{\mu \nu}+f_{2134}^{\mu \nu}=0, \quad f_{1234}^{\mu \nu}+f_{3124}^{\mu \nu}+f_{2314}^{\mu \nu}=0, \\
f_{1234}^{\mu \nu}-f_{1243}^{\mu \nu}+f_{3412}^{\mu \nu}-f_{3421}^{\mu \nu}=0 .
\end{gathered}
$$

The corresponding current is given by

$$
\begin{aligned}
\mathcal{F}_{1234}^{\mu \nu}= & \frac{1}{s_{1234}}\left(\frac{f_{1234}^{\mu \nu}}{s_{12} s_{123}}+\frac{f_{3214}^{\mu \nu}}{s_{23} s_{123}}+\frac{f_{1234}^{\mu \nu}-f_{1243}^{\mu \nu}}{s_{12} s_{34}}\right. \\
& \left.-\frac{f_{4321}^{\mu \nu}}{s_{34} s_{234}}-\frac{f_{2341}^{\mu \nu}}{s_{23} s_{234}}\right) .
\end{aligned}
$$

\section{Five-particle case}

See the Supplemental Material [36] for the five-particle polarizations, field-strength tensors and their GJI.

\section{BUILDING AMPLITUDES WITH MULTIPARTICLE CYCLES AND TAILS}

The trees that we have found can be attached back to the propagator to compute tree-level amplitudes with colorkinematics duality, or we can just use the current numerators to build local BCJ numerators for the color-ordered amplitudes (for this type of procedures in tree-level amplitudes and examples see e.g., [7,37]).

But let us now return to the one-loop gluon amplitudes. Our calculations above have taught us that the pinching of the ordinary Lorentz cycles produces smaller cycles with insertions of multiparticle field-strength tensors, and the pinching of tails leads to lower-point tails involving multiparticle polarizations. Moreover, for the cycle part such 
insertions have already shown to be useful and natural for various amplitudes in SYM theory; generalized two-cycles already appeared in six-dimensional SYM in $[8,9]$, there called "scalar fundamentals", and a multiparticle version of the $t_{8}$ tensor, which in $\mathcal{N}=4 \mathrm{SYM}$ absorbs the tensor structure of the four-gluon amplitude, was introduced in [12].

This leads us to conjecture that the complete effect of the pinching procedure in the Bern-Kosower formalism may, in the $Q_{n}$ integrand, be taken into account simply by adding to the unpinched integrand all combinations of generalized cycles and tails that are possible for the given number of gluons, and compatible with the fixed color ordering. Thus we now define a new generalized Lorentz cycle

$$
\mathcal{Z}_{k}\left(I_{1}, \ldots, I_{k}\right) \equiv\left(\frac{1}{2}\right)^{\delta_{k 2}} \operatorname{tr}\left(\prod_{i=1}^{k} \mathcal{F}_{I_{i}}\right)
$$

which, contrary to (1), now uses the full Berends-Giele currents $\mathcal{F}_{I_{i}}$, and the generalized tail

$$
\mathcal{T}_{k}\left(I_{1}, \ldots, I_{k}\right) \equiv T\left(k_{I_{1}}, \mathcal{A}_{I_{1}} ; \ldots ; k_{I_{k}}, \mathcal{A}_{I_{k}}\right)
$$

For example, the three-gluon amplitude, whose unpinched integrand is (5), should have the pinch contribution

$$
\dot{G}(1,23)+\dot{G}(2,31)+\dot{G}(3,12)
$$

where, e.g.,

$$
\dot{G}(1,23)=\dot{G}_{12} \dot{G}_{21} \frac{1}{2} \operatorname{tr}\left(f_{1} \mathcal{F}_{23}\right)
$$

and this is indeed what the Bern-Kosower pinch rules produce. Similarly, at the four-point level the prediction for the single-pinch terms would be

$\dot{G}(1,2,34)+\dot{G}(1,23) T(4)+\dot{G}(1,2) \mathcal{T}(34)+$ perm.

and for the double pinches,

$$
\dot{G}(1,234)+\dot{G}(12,34)+\text { perm } .
$$

which we have again found to be in agreement with the application of the pinch rules.

This result for the double pinches can be straightforwardly generalized to the maximal, i.e., $(n-2)$-fold, pinch contribution to the $n$-gluon amplitude. This is because the result of such a pinch can only be a bubble diagram, and the bubbles can always be represented as a Lorentz two-cycle times a $\dot{G}_{i j}^{2}$, as we saw in (15) and have used above. For the bubble integrands we then have the compact expression for a given ordering $I$, namely

$B_{n}^{I}=\sum_{\substack{I=J K \\|I|, \mid J \geq 2}} \sum_{\sigma \in \operatorname{cyc}(I)} \mathcal{Z}_{2}\left(\sigma_{j_{1}} \cdots \sigma_{j_{n-l}}, \sigma_{k_{n-l+1}} \cdots \sigma_{k_{n}}\right) \dot{G}_{\sigma_{j_{1}} \sigma_{k_{n-l+1}}}$,

where the first sum accounts for the deconcatenation of the word $I$ into words $J$ and $K$ of length bigger than one.

\section{SUMMARY AND OUTLOOK}

We have presented a novel method of constructing Berends-Giele currents using the information on these contained in the pinch procedure of the Bern-Kosower formalism. The method comes in two versions, cycle pinching vs tail pinching, based on two specific pinch contributions to the one-loop $n$-gluon amplitudes. Explicit calculation up to the five-point case has shown that the multiparticle fields numerators of these currents obey the GJI required by BCJ gauge, indicative of color-kinematics duality. Our results also indicate that the tail-pinching approach becomes superior at higher points since it leads directly and unambiguously to BCJ gauge multiparticle polarizations without the usual addition of judiciously chosen $h$-scalars. We are currently working on a formal proof for the all- $n$ case, based on our understanding of the general structure of the polynomials presented here.

A connection between the Bern-Kosower formalism and color-kinematics duality was previously hinted at in [38] where it was shown that all the Bern-Kosower numerators satisfy BCJ identities through a detailed analysis of the field-theory limit of the monodromy relations of string theory at one loop; it would be interesting to compare their results with ours.

We have further shown that using these Berends-Giele currents as words in generalized Lorentz cycles, and the associated multiparticle polarization vectors in generalized tails, provides an extremely attractive approach towards absorbing the effect of the Bern-Kosower pinching procedure into multiparticle tensor structures. We hope to obtain (along these lines) a representation of the one-loop $n$-gluon amplitudes that would be ultracompact as well as exhibit manifest color-kinematics duality.

\section{ACKNOWLEDGMENTS}

We thank Renann Lipinski Jusinkas, Carlos Mafra and Oliver Schlotterer for carefully reading the manuscript. C. L. A. thanks Carlos Mafra and Oliver Schlotterer for fruitful discussions and clarifications in comparing our results with theirs. 
[1] N. Ahmadiniaz and C. Schubert, Europhys. Lett. 130, 41001 (2020).

[2] F. A. Berends and W. T. Giele, Nucl. Phys. B306, 759 (1988).

[3] S. Lee, C. R. Mafra, and O. Schlotterer, J. High Energy Phys. 03 (2016) 090.

[4] Z. Bern, J. J. M. Carrasco, and H. Johansson, Phys. Rev. D 78, 085011 (2008).

[5] C. R. Mafra and O. Schlotterer, J. High Energy Phys. 07 (2014) 153.

[6] C. R. Mafra and O. Schlotterer, Phys. Rev. D 92, 066001 (2015).

[7] C. R. Mafra and O. Schlotterer, J. High Energy Phys. 03 (2016) 097.

[8] M. Berg, I. Buchberger, and O. Schlotterer, J. High Energy Phys. 04 (2017) 163.

[9] M. Berg, I. Buchberger, and O. Schlotterer, J. High Energy Phys. 07 (2017) 138.

[10] L. M. Garozzo, L. Queimada, and O. Schlotterer, J. High Energy Phys. 02 (2019) 078.

[11] E. Bridges and C. R. Mafra, J. High Energy Phys. 10 (2019) 022.

[12] A. Edison, S. He, O. Schlotterer, and F. Teng, J. High Energy Phys. 09 (2020) 079.

[13] H. Kawai, D. C. Lewellen, and S. H. H. Tye, Nucl. Phys. B269, 1 (1986).

[14] Z. Bern, J. J. M. Carrasco, and H. Johansson, Phys. Rev. Lett. 105, 061602 (2010).

[15] Z. Bern and D. A. Kosower, Phys. Rev. Lett. 66, 1669 (1991).

[16] Z. Bern and D. A. Kosower, Nucl. Phys. B362, 389 (1991).

[17] Z. Bern and D. A. Kosower, Nucl. Phys. B379, 451 (1992).

[18] M. J. Strassler, Nucl. Phys. B385, 145 (1992).

[19] M. J. Strassler (unpublished).
[20] C. Schubert, Phys. Rep. 355, 73 (2001).

[21] J. P. Edwards and C. Schubert, arXiv:1912.10004.

[22] M. G. Schmidt and C. Schubert, Phys. Rev. D 53, 2150 (1996).

[23] N. Ahmadiniaz, C. Lopez-Arcos, M. A. Lopez-Lopez, and C. Schubert, arXiv:2012.11791.

[24] N. Ahmadiniaz and C. Schubert, Nucl. Phys. B869, 417 (2013).

[25] N. Ahmadiniaz and C. Schubert, Int. J. Mod. Phys. E 25, 1642004 (2016).

[26] C. Schubert, Eur. Phys. J. C 5, 693 (1998).

[27] A. M. Garsia, in Analysis, et Cetera, edited by P. H. Rabinowitz and E. Zehnder (Academic Press, New York, 1990), pp. 309-382.

[28] C. Reutenauer, Free Lie Algebras, LMS Monographs (Clarendon Press, Oxford, 1993).

[29] Z. Bern, L. J. Dixon, M. Perelstein, and J. S. Rozowsky, Nucl. Phys. B546, 423 (1999).

[30] N. E. J. Bjerrum-Bohr, P. H. Damgaard, T. Sondergaard, and P. Vanhove, J. High Energy Phys. 01 (2011) 001.

[31] C. R. Mafra, J. High Energy Phys. 07 (2016) 080.

[32] C. R. Mafra, arXiv:2011.14413.

[33] H. Frost, C. R. Mafra, and L. Mason, arXiv:2012.00519.

[34] S. Mizera and B. Skrzypek, J. High Energy Phys. 10 (2018) 018.

[35] C. Lopez-Arcos and A. Q. Vélez, J. High Energy Phys. 11 (2019) 010.

[36] See Supplemental Material at http://link.aps.org/ supplemental/10.1103/PhysRevD.104.L041702 for more details.

[37] H. Gomez, R. L. Jusinskas, C. Lopez-Arcos, and A. Q. Vélez, J. High Energy Phys. 02 (2021) 093.

[38] E. Casali, S. Mizera, and P. Tourkine, J. High Energy Phys. 03 (2021) 048. 